\title{
Ocorrência do gênero Campsurus (Ephemeroptera, Polymitarcyidae) no Semi-Árido do Estado do Rio Grande do Norte, Brasil 1
}

\author{
Bianca Maira Paiva Ottoni², Marcos Paulo Gomes Pinheiro², Herbet Tadeu de Almeida Andrade² \\ \& Ricardo Andreazze 2
}

1. Projeto financiado pelo CNPq. 2. Universidade Federal do Rio Grande do Norte, e-mail: biancaottoni@gmail.com (Autor para correspondência), marcospinheiro.pinheiro@bol.com.br, herbet@ufrnet.br, andreazze@cb.ufrn.br

EntomoBrasilis 2 (3): 90-91 (2009)

Resumo. Este estudo relata, pela primeira vez, a ocorrência do gênero Campsurus para o Semi-Árido do estado do Rio Grande do Norte, Brasil.

Palavras-chave: Campsurus, Polymitarcyidae, Rio Grande do Norte, Semi-árido.

\section{The Occurrence of the Genus Campsurus, (Ephemeroptera, Polymitarcyidae) in Semi-Arid of the State of Rio Grande do Norte, Brazil.}

Abstract. This study reports for the first time the occurrence of the genus Campsurus in the Semi-arid of the state of Rio Grande do Norte, Brazil.

Keywords: Campsurus, Polymitarcyidae, Rio Grande do Norte, Semi-arid.

A ordem Ephemeroptera (Insecta) está representada por organismos anfibióticos, com adultos de hábito terrestre e imaturos aquáticos que ocorrem praticamente em todos os tipos de corpos d'água (rios, lagos, brejos e fitotelmatas) (DIAS et al. 2007), possuindo hábitos alimentares do tipo saprófago ou herbívoro (McCAFFERTY \& PROVONSHA 1994).

Autores como CROWL et al. (1997) e ROSENFELD (1997) citam as ninfas de efemerópteros como parte importante da dieta de peixes em variados ambientes aquáticos. Além disso, estudos feitos por NOLTE (1987) e WALKER (1995) demonstraram a importância dessa ordem para os ecossistemas aquáticos, especialmente no que se refere à produção secundária, ciclo de nutrientes, processo de decomposição da matéria orgânica, bioturbação e como biondicadores da qualidade da água.

Atualmente, são conhecidas 14 famílias de Ephemeroptera, compreendendo aproximadamente 4000 espécies, sendo conhecidas 450 na América do Sul (DOMINGUEZ et al. 2006). No Brasil existem 63 gêneros conhecidos de Ephemeroptera, divididos em 10 famílias (SALLES et al. 2004).

A família Polymitarcyidae não está entre as mais numerosas da ordem, no entanto, seu gênero Campsurus é considerado o mais diverso, possuindo distribuição Neoártica e Neotropical (DOMINGUEZ et al. 2006), com 42 espécies descritas para a América do Sul e 24 para o Brasil (SALLES 2006).

SALLES et al. (2004) relatam a ocorrência do gênero Campsurus nos estados: Amazonas, Pará, Matos Grosso do Sul, Minas Gerais, Espírito Santo, Rio de Janeiro, São Paulo, Santa Catarina e Rio Grande do Sul; já BRITO-JÚNIOR et al. (2005) relataram a ocorrência de Campsurus sp. no município de São José dos Cordeiros no semi-árido do estado da Paraíba.

No Estado do Rio Grande do Norte, embora existam trabalhos utilizando macroinvertebrados bentônicos em rios do semi-árido, tais como MELO-JÚNIOR \& IRUSTA (2004); IDEMA (2007) e ANDRADE et al. (2008), a família Polymitarcyidae jamais havia sido encontrada. Deste modo, visando contribuir para a expansão do conhecimento sobre a entomofauna bentônica do semi-árido potiguar, este estudo constitui o primeiro registro de ocorrência para a essa região, tanto dessa família como de seu gênero Campsurus.

As coletas foram realizadas ao longo do trecho baixo do Rio Piranhas-Açu, em quatro pontos amostrais, localizados nas proximidades dos municípios Açu, Ipanguaçu, Alto do Rodrigues e Pendências, todos inseridos na região do semi-árido do Rio Grande do Norte, nordeste do Brasil. Foram executadas seis amostragens mensais de sedimento, três no período de seca (outubro a dezembro de 2007) e três no período de chuva (março a maio de 2008), através de draga de Van Veen com $0,377 \mathrm{~cm}^{2} \mathrm{de}$ área, de acordo com BICUDO \& BICUDO (2004).

Todo o material coletado foi transportado do campo para o Laboratório de Entomologia da Universidade Federal do Rio Grande do Norte (LABENT) em frascos contendo álcool $80 \%$. No LABENT, houve a triagem, a contagem e a identificação dos exemplares de acordo com as chaves proposta por SALLES (2006). Os exemplares coletados foram depositados na coleção entomológica da Universidade Federal do Rio Grande do Norte.

Foi coletado um total de 1808 exemplares da ordem Ephemeroptera, desses 986 foram identificados como Polymitarcyidae, todos do gênero Campsurus.

Os espécimes do gênero foram coletados apenas nos meses de outubro, novembro e dezembro de 2007 e em março de 2008, somente nos pontos de Alto Rodrigues com um total de 954 exemplares e Pendências com 32, não havendo assim, registros nos outros pontos amostrais. Possivelmente, essa ausência está relacionada com as características do sedimento e da qualidade da água dos pontos de Açu e Ipanguaçu, estudos mais aprofundados, considerando dados físicos e químicos e 
análises estatísticas convenientes, devem ser realizados para a confirmação dessa suposição.

Baseando-se em SALLES et al. (2004) este é o primeiro registro do gênero Campsurus para o Estado do Rio Grande do Norte, aumentando o conhecimento sobre a biodiversidade bentônica da região e sendo mais um alicerce de pesquisas de biomonitoramento da qualidade da água através de macroinvertebrados bentônicos e de futuros estudos limnológicos sobre bioturbação utilizando esses organismos em rios do semiárido do nordeste brasileiro.

\section{AGRADECIMENTOS}

À Prof ${ }^{\mathrm{a}}$ Dr ${ }^{\mathrm{a}}$ Maria José Nascimento Lopes do Instituto Nacional de Pesquisas da Amazônia (INPA) pela valorosa contribuição, confirmando a identificação do gênero. Ao Conselho Nacional de Desenvolvimento Científico e Tecnológico (CNPq) e à Coordenação de Aperfeiçoamento de Pessoal de Nível Superiora (CAPES) pela concessão das bolsas de mestrado, respectivamente, ao primeiro e segundo autor. Este trabalho é resultado do Projeto Insetos Bentônicos Bioindicadores no Rio Piranhas-Açu - Área de Ação Petrolífera do Estado do Rio Grande do Norte, CNPq no 555377/2006-7.

\section{REFERÊNCIAS}

Andrade, H.T.A., A.S. Santiago \& J.F. Medeiros, 2008. Estrutura da Comunidade de Invertebrados Bentônicos com Enfoque nos Insetos Aquáticos do Rio Piranhas-Assu, Rio Grande do Norte, Nordeste do Brasil. EntomoBrasilis 1(3): 51-56.

Bicudo, C.E.M. \& D.C. Bicudo, 2004. Amostragem em Limnologia. São Carlos/SP: RiMa. 371p.

Brito-Júnior, L., F.J.P. Abílio \& T. Watanabe, 2005. Insetos aquáticos do açude São José dos Cordeiros (semi-árido paraibano) com ênfase em Chironomidae. Entomologia y Vectores. 12:149-157.

Crowl, T.A., C.R. Townsend, N. Bouwes \& H. Thomas, 1997. Scales and causes of patchiness in stream invertebrate assemblages: top-down predator effects? Journal of the North American Benthological Society. 16:277-285.

\section{Como citar este artigo:}

Ottoni, B.M.P, M.P.G. Pinheiro, H.T.A. Andrade \& R. Andreazze, 2009. Ocorrência do gênero Campsurus (Ephemeroptera, Polymitarcyidae) no Semi-Árido do Estado do Rio Grande do Norte, Brasil. EntomoBrasilis, 2(3): 90-91. www.periodico.ebras.bio.br/ojs
Dias, L.G., F.F. Salles, C.M. Polegatto, R.S. Mariano \& C.G. Froehlich, 2007. Novos registros de Ephemerelloidea (Insecta: Ephemeroptera) para o estado de São Paulo. Biota Neotropica (Ed. Portuguesa), v. 7, p. 37-40.

Dominguez, E., C. Molineri, M. Pescador, M.D. Hubbard \& C. Neto, 2006. Aquatic Biodiversity in Latin América, Vol. 1: Ephemeroptera of South América. Pensoft.

IDEMA - Instituto de Defesa do Meio Ambiente do Rio Grande do Norte, 2007. Sistema de indicadores e índices ambientais para o RN: Bacia Potiguar, Relatório Técnico. (Diniz, R.F., L.P. Brito, J.D. Tinôco, M.V.S. Souto, J.B. Irusta, S.L. Oliveira \& H.T.A. Andrade) (eds). 322pp.

McCafferty, W.P. \& A. Provonsha, 1994. Aquatic Entomology. Jones and Bartlett Publishers, Inc., Boston.

Melo-Júnior, G., J.B. Irusta, 2004. Relatório do Projeto "Diagnóstico e Monitoramento da Área de influência do Projeto Termoaçu - Município de Alto do Rodrigues, Rio Grande do Norte $2^{\text {a }}$ fase". $542 \mathrm{p}$.

Nolte, U., 1987. Campsurus notatus (Polymitarcidae, Ephemeroptera) a bioturbator in Várzea lakes. Amazoniana 10: 219-222.

Rosenfeld, J.S., 1997. The influence of upstream predation on the expression of fish effects in downstream patches. Freshwater Biology 37: 535-543.

Salles, F.F., E.R. Da-Silva, M.D. Hubbard \& J.E. Serrão, 2004. As espécies de Ephemeroptera (Insecta) registradas para o Brasil. Biota Neotropica. 4:1-34.

Salles, F.F., 2006. A ordem Ephemeroptera no Brasil (Insecta): taxonomia e diversidade. Tese de Doutorado apresentada ao Programa de Pós-Graduação em Entomologia da Universidade Federal de Viçosa. 300 p.

Walker, I., 1995. Amazonian streams and small rivers. In: Tundisi, J. G., C. E. M. Bicudo \& T. M. Tundisi (eds), Limnology in Brazil. ABC/SBL, Rio de Janeiro: 167-193.

Recebido em: 12/05/2009

Aceito em: 24/08/2009

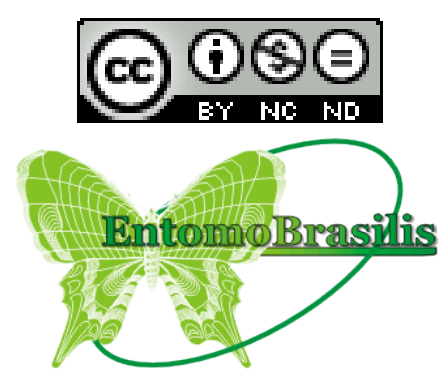

